\title{
Perspectivas teóricas para a análise de políticas públicas: como lidam com a complexidade?
}

\author{
Frameworks for public policy analysis: how do they deal with complexity?
}

Perspectivas teóricas para el análisis de políticas públicas: cómo lidian con la complejidad?

Lia de Azevedo Almeida

Doutorado em Administração pela Universidade de Brasília Professora Adjunta da Universidade Federal do Tocantins http://lattes.cnpq.br/7835915125417077 https://orcid.org/0000-0002-6586-4067

lia.almeida@uft.edu.br

Ricardo Corrêa Gomes

Pós-doutor pela Georgia State University (2014), Doutor em Gestão Pública pela Aston Business School (2003), Professor Associado III da Universidade de Brasília http://lattes.cnpq.br/3539564256173485 gomesric.rg@gmail.com

Resumo: O presente ensaio buscou comparar as perspectivas teóricas próprias da análise de políticas públicas de meados dos anos 1980 e início dos anos 1990, com as novas teorias da área, buscando identificar diferenças ou semelhanças quanto à incorporação de aspectos próprios da teoria da complexidade e dos sistemas complexos, especificamente os conceitos de emergência, equilíbrio dinâmico, adaptação e co-evolução. Dentre os modelos tradicionais, optou-se por comparar os modelos considerados consagrados (o modelo de múltiplos fluxos, o de equilíbrio pontuado e o de coalizões de advocacia) com as novas teorias da área que assumem explicitamente a complexidade do processo político: o modelo de Ecologia de Jogos e o modelo de Robustez. Os modelos tradicionais tocam na complexidade de forma tangencial, utilizando-se de alguns conceitos como uma metáfora para explicar determinadas situações do processo político, como a ascensão de temas à agenda e o aprendizado político. Nas novas teorias analisadas, a interdependência dinâmica entre agente e estrutura aparece mais explicitamente. Se comparado ao modelo de Robustez, o modelo de Ecologia dos Jogos parece mais completo, incorporando os principais conceitos do sistema complexo, como equilíbrio dinâmico, emergência, adaptação, co-evolução. As diferenças entre os modelos novos e os tradicionais, tem razões explicativas no próprio entendimento do que vem a ser o processo político e do papel que a análise de políticas públicas deve cumprir, se uma função mais prescritiva ou descritiva.

Palavras-chave: análise de políticas públicas, modelos teóricos, teoria da complexidade, sistemas complexos.

\begin{abstract}
This study sought to compare the theoretical perspectives of public policy analysis of the mid-1980s and early 1990s with the new theories in the field, seeking to identify differences or similarities regarding the incorporation of specific aspects of complex theory and complex systems, specifically concepts of emergence, dynamic equilibrium, adaptation, and $\mathrm{co}-$ evolution. Among the traditional frameworks, we opt to compare the established frameworks (the Multiple Streams model, Punctuated Equilibrium, and Advocacy Coalitions) with the new theories that explicitly assume the complexity of the policy process, namely, the Ecology of Games Framework and the Robustness Framework.Traditional frameworks address complexity in a tangential way, using some concepts as a metaphor to explain certain situations in the policy process, such as the rise of themes to the agenda and policy learning. In the new analyzed frameworks, the dynamic interdependence between agent and structure appears more explicit. Compared to the Robustness Framework, the Ecology of Games seems more complete, incorporating the main concepts of a complex system, such as dynamic equilibrium, emergence, adaptation, and coevolution. The differences between the new and traditional models offer explanations for the very understanding of what the policy process is and the role that policy analysis must fulfill, be it a more prescriptive or descriptive function.
\end{abstract}

Keywords: public policy analysis, policy frameworks, complexity theory, complex systems
Resumen: El presente ensayo buscó comparar las perspectivas teóricas propias del análisis de políticas públicas de mediados de los años 1980 y principios de los años 1990, con las nuevas teorías del área, buscando identificar diferencias o semejanzas en cuanto a la incorporación de aspectos propios de la teoría de la complejidad y de los sistemas complejos, específicamente los conceptos de emergencia, equilibrio dinámico, adaptación y co-evolución. Entre los modelos tradicionales, se optó por comparar los modelos considerados consagrados (el modelo de múltiples flujos, el de equilibrio puntuado y el de coaliciones de abogacía) con las nuevas teorías del área que asumen explícitamente la complejidad del proceso político: el modelo de múltiples, Ecología de Juegos y el modelo de Robustez. Los modelos tradicionales tocan en la complejidad de forma tangencial, utilizando algunos conceptos como una metáfora para explicar determinadas situaciones del proceso político, como el ascenso de temas a la agenda y el aprendizaje político. En las nuevas teorías analizadas, la interdependencia dinámica entre agente y estructura aparece más explícitamente. Si se compara al modelo de Robustez, el modelo de Ecología de los Juegos parece más completo, incorporando los principales conceptos de sistema complejo, como equilibrio dinámico, emergencia, adaptación, co-evolución. Las diferencias entre los modelos nuevos y los tradicionales, tienen razones explicativas en el propio entendimiento de lo que viene a ser el proceso político y del papel que el análisis de políticas públicas debe cumplir, si una función más prescriptiva o descriptiva.

Palabras clave: análisis de políticas públicas, modelos teóricos, teoría de la complejidad, sistemas complejos.

Texto completo em português: http://www.apgs.ufv.br

Full text in Portuguese: http://www.apgs.ufv.br

\section{Introdução}

Atualmente, há um entendimento comum de que as políticas públicas seriam formuladas por arranjos, com graus variados de abrangência, interdependência e compromisso, caracterizados pela interação de diferentes tipos de atores, o que pode ser percebido a partir dos vários conceitos que têm sido discutidos na literatura recente, como subsistemas de políticas públicas, redes, comunidades de políticas públicas, comunidades epistêmicas, governança, entre outros.
Sabatier (2007, p. 8) reconhece essa complexidade ao afirmar que "o processo político envolve uma vasta gama de elementos extremamente complexos que interagem ao longo do tempo". Uma série de relações é subjacente a esse fenômeno, como: "o conhecimento dos objetivos e percepções de muitos atores, o papel das instituições decisórias, relações de poder, ideias, valores e tempo" (Sabatier, 2007, p.8). É a partir desse processo político que as políticas públicas emergem e são moldadas ao longo do tempo. 
Sabatier e Jekins-Smith (1993;1999) desenvolveram o modelo de coalizões de advocacia no início da década de 1990, buscando justamente uma teorização mais abrangente que pudesse incorporar essas relações, fazendo uma crítica ao modelo de estágios de Lasswell (1951) (Sabatier, 2007; Weible, Sabatier \& Mcqueen, 2009).

Em meados de 1980 e início dos anos 1990, surgiram alguns modelos que contrapunham a visão sequencial ou de estágios: o modelo de Múltiplo Fluxos (Multiples Streams) proposto por John Kingdon em 1984, o de Coalizões de Advocacia (Advocacy Coalitions Framework) proposto por Paul Sabatier e Jekins-Smith em 1993 (posteriormente revisado e ampliado pelos autores em 1999) e o modelo de Equilíbrio Pontuado (Punctuated Equilibrium) elaborado por Frank Baumgartner e Brian Jones em 1993.

Em comum, os três modelos, entendem o processo político pelo qual originam as políticas públicas, como complexo, instável, e sujeito a relações de poder entre diversos atores. Além disso, incluem importantes variáveis em suas análises, como as ideias, a mídia, e a opinião pública, considerando-as importantes influências na consolidação de uma política.

Entretanto, devido ao alto grau de complexidade inerente ao processo político, recentemente, alguns autores têm criticado esses modelos pela sua impossibilidade de compreender tal processo de forma holística, integrando ao mesmo tempo as dimensões de agencia e estrutura (Morçöl, 2010; Budd, Charlesworth \& Paton, 2006 ; Ferlie, Lynn \& Pollitt, 2005; Goodin, Rein \& Moran, 2006), e também pelo fato de privilegiar a explicação da estabilidade do processo político ao invés de sua instabilidade (Eppel, 2016, 2009; Haynes, 2009; 2008).

Morçöl (2010) chama atenção para o fato de que os modelos tradicionais $^{12}$ de políticas públicas (aqueles mencionados por Sabatier (2007), e dentre os quais encontram-se o modelo de coalizões de advocacia, múltiplos fluxos e equilíbrio pontuado), não conseguem superar a dicotomia entre agência e estrutura, argumentando que é necessária uma visão que transcenda a dualidade entre micro/macro, ou seja, entre agência e estrutura, à semelhança da teoria da estruturação de Giddens (1984). Neste sentido, Haynes (2009) defende a necessidade de ter uma visão de "helicóptero", que proporcione uma vista panorâmica, que possibilite voltar o olhar também para a estrutura, que segundo ele, torna-se tão importante quanto à análise do comportamento micro. Outra crítica dirigida aos modelos tradicionais, é o fato de que ainda são estruturados em uma causalidade linear, e que as pesquisas ainda estão mais propensas a enfatizar a relativa estabilidade do processo político, ao invés de sua relativa instabilidade (Haynes, 2009).

Paralelamente às críticas, estudos recentes têm alertado para o potencial de contribuição da teoria da complexidade para o campo de políticas públicas, como forma de alcançar uma visão que não

${ }^{1}$ Adota-se, aqui, a terminologia processo político, em sintonia com a literatura internacional que utiliza a expressão "policy process", que poderia ser traduzido como "processo político da política pública". Para simplificar será utilizado termo "processo político" para se referir ao processo pelo qual as políticas públicas são construídas. priorizaria agência ou estrutura ou instabilidade e estabilidade do processo (Morçöl, 2012, 2010, 2005; Eppel, 2016, 2009; Haynes, 2009). Adicionalmente, há discussões apontando que a teoria da complexidade pode contribuir para a área de políticas públicas de forma geral, embora com algumas ressalvas, pois não há consenso sobre a própria teoria da complexidade. Isto se deve ao fato de que que existem trabalhos diferentes aglutinados sob o mesmo leque da complexidade, como ressalta Cairney (2012, p.354), que questiona se existe uma teoria da complexidade ou se "devemos falar de muitas teorias da complexidade?". A mesma resslava também aparece em Geyer e Rihani (2010), que afirmam que "a teoria complexidade pode complicar o estudo das políticas públicas sem oferecer algo novo ou seus insights podem ser descritos como ‘óbvios e de senso comum”' (Geyer \& Rihani, 2010, p.186).

Adicionalmente, outros autores têm alertado para o seu potencial de aplicação para vários campos da administração pública, incluindo ou não os processos de políticas públicas, como em Koppenjan e Klijn (2014), Koliba, (2014), Gerrits e Marks (2015), Rhodes et al. (2011), Teisman, van Buuren e Gerrits (2009), Teisman e Klijn (2008), e Klijn, (2008).

O objetivo deste ensaio é identificar em que medida os conceitos da teoria da complexidade e sistemas complexos são incorporados nas perspectivas teóricas próprias da análise de políticas públicas, comparando-se o movimento teórico de meados dos anos 1980 e início dos anos 1990, com as novas teorias, buscando identificar diferenças ou semelhanças. Com isso, buscase responder o seguinte questionamento: Em que medida os modelos de análise de políticas públicas têm incorporado aspectos da teoria da complexidade para compreender o processo político caracterizado por um ambiente de incerteza e cada vez mais complexo?

Verificar como as novas teorias responderam ao chamamento dos próprios acadêmicos que afirmam que a complexidade poderia auxiliar nos estudos de políticas públicas, é uma contribuição deste artigo para o campo de análise de políticas públicas. Espera-se, com essa análise, situar a evolução da utilização dos conceitos da complexidade nas teorias de políticas públicas de forma a proporcionar subsídios para que se amplie a própria discussão sobre análise de políticas públicas.

Para a consecução do objetivo, este ensaio foi estruturado em cinco seções. Primeiramente, é feita uma revisão sobre a teoria da complexidade e seus principais conceitos. Na segunda parte, temse uma breve revisão sobre a evolução da teorização do processo político, que passa de um processo decisório de caráter técnico, marcado por etapas, até o entendimento como um processo complexo. Em seguida, discute-se em que medida os três modelos desenvolvidos em meados da década de 1980 e início dos anos 1990, o de Múltiplos Fluxos (MF), Coalizões de Advocacia (ACF) e Equilíbrio Pontuado (EP), incorporaram em suas estruturas

2 É importante frisar que neste artigo quando houver a referencia à "modelos tradicionais", entende-se aqueles mencionados por Sabatier (2007): o modelo institucional da escolha racional, múltiplos fluxos, equilíbrio pontuado, coalizões de advocacia, análise de redes e construção social, conforme entendimento de Morçol (2010). 
conceitos próprios da teoria da complexidade. Na quarta seção, discute-se como as perspectivas teóricas mais recentes, o modelo de Robustez e de Ecologia de Jogos (EG) trabalham com a questão da complexidade, e em que medida utilizam seus conceitos e metodologias. Por fim, apresenta-se as diferenças ou semelhanças quanto as duas gerações teóricas, no que tange a incorporação de aspectos da teoria da complexidade, buscando refletir sobre o estado do campo teórico em políticas públicas.

\section{Teoria da complexidade e sistemas complexos}

O objetivo dessa seção é apresentar o conceito de sistema complexo e suas características, pois, conforme discutido na introdução, autores argumentam que a teoria da complexidade pode colaborar no estudo de políticas públicas, pelo fato de que o processo político do qual originam as políticas públicas é, de fato, um sistema complexo. Portanto, essa seção busca levantar os principais conceitos de sistema complexo para que se possa analisar nas seções seguintes como estes foram incorporados explícita ou implicitamente nas teorias tradicionais e nas novas teorias da área.

Embora a ideia de uma teoria da complexidade não seja considerada como plenamente desenvolvida para que alcance o status de uma teoria, há inegavelmente uma série de teorias e conceitos relacionados a um pensamento complexo, que é aplicado em diversas áreas do conhecimento. Mitleton-Kelly (2003, apud Klijn 2008, p.301) distingue cinco diferentes áreas de pesquisa abarcadas sobre o rótulo da complexidade: os sistemas complexos adaptativos, originários dos trabalhos de Kauffman (1990), estruturas dissipativas, originárias dos trabalhos de Prigogine (1996), a teoria da autopoiese (Maturana \& Varela, 1991), teoria do caos.

As críticas a respeito da validade da teoria da complexidade variam entre os argumentos de que não há uma unificação da teoria, com um conjunto organizado proposições testáveis (Horgan, 1995), até as afirmações de que constituem mais um conjunto de conceitos ferramentais do que um corpo teórico definitivo (Bousquet \& Curtis, 2011). De forma geral, todas as teorias englobadas sob o rótulo da Complexidade estão interessadas em como a natureza de um sistema pode ser caracterizada a partir de suas partes constituintes de uma maneira não reducionista (Manson, 2001), aceitando a desordem, a incerteza e a imprevisibilidade (Goulielmos, 2005).

Quanto à definição de sistemas complexos, Casti (1994) afirma que são aqueles tipos de sistema que possuem as "impressões digitais" da complexidade. É importante destacar que há muitas definições diferentes de um sistema complexo, mas em geral ele possui os seguintes elementos: (i) vários agentes heterogêneos; (ii) os agentes agem localmente e possuem racionalidade limitada; (iii) os agentes interagem entre si, o que altera o sistema (emergência de padrões no nível macro que não podem ser previstos a partir do comportamento individual); (iv) não há necessariamente equilíbrio, e sim um processo dinâmico de mudança constante com possíveis períodos mais estáveis; (v) os agentes alteram seu comportamento em resposta do padrão emergente; (vi) ocorre um processo evolucionário, que seleciona ou elimina os padrões emergentes (Wu \& Marceau, 2002; Cilliers, 2005; Holland, 1995; Prigogine, 1987);

Desta forma, conceitos como: não linearidade (consequência das propriedades emergentes), adaptação e co-evolução são conceitos essenciais para o se compreender o comportamento de um sistema complexo. Cada uma dessas características serão explicadas a seguir.

As propriedades emergentes são as mais evidentes características que distinguem um sistema complexo de um sistema simples, e que explicam a não linearidade, a imprevisibilidade e impossibilidade de controle de um sistema complexo. Isto significa dizer que podem surgir mudanças abruptas a partir de ações simples de partes do sistema. A emergência está ligada à ideia de um "todo maior do que a soma das partes" (Morin, 2003), ou seja, a interação dos agentes no sistema forma padrões de organização mais complexos do que os padrões dos próprios agentes (Agar, 1999). Além disso, a emergência existe em razão da interação entre os componentes do sistema, e não da simples sobreposição entre as partes.

A emergência é exemplificada por Baranger (2000) da seguinte forma: o corpo humano é capaz de caminhar, o que é uma propriedade emergente. Contudo, estudando-se somente a cabeça, somente o tronco ou somente os membros de uma pessoa, não haverá a compreensão da capacidade de caminhar. É a propriedade emergente dos sistemas complexos que impossibilita sua previsão e controle, uma vez que mudanças em um subsistema visando à mudança do todo podem não obter o resultado esperado, já que os outros componentes do sistema mudam para se adaptar à intervenção, além de outras mudanças no ambiente (Manson, 2001).

Associado ao conceito de emergência, está o conceito de feedback, na medida que seu papel é de particular importância, uma vez que se trata de relacionamentos não lineares entre entidades em constante mudança (Manson, 2001). Diferentemente da teoria geral dos sistemas, em que a noção de feedback está ligada a linearidade, ou seja, uma mudança em A causa uma mudança proporcional em $\mathrm{B}$, no sistema complexo o fato de as partes do sistema interagir gerando feedbacks não intencionais e em diferentes escalas, é justamente o fator que a explica a mudança não linear, ou seja, uma mudança em $\mathrm{A}$ pode ter efeitos imprevisíveis.

Quanto à adaptação, esta é outra característica marcante dos sistemas complexos, inclusive muitos autores utilizam a denominação de "sistemas complexos adaptativos" (Stacey 1996; Holland,1995, Cohen \& Axerold, 2000, Leite, 2004, Allison, 1998; Daft, 2002, Gell-mann, 1996), isso porque estes sempre buscam um padrão. Eles interagem com o meio ambiente, "aprendem" com a experiência e como resultado se adaptam (Gell-man, 1996).

Segundo Stacey (1996), a interação e o aprendizado dos agentes em sistemas acontecem individualmente, por meio de um processo que envolve descoberta, escolhas e ação. Os agentes possuem "autonomia para orientarem suas ações de acordo com o que aprendem de sua interação com o ambiente - o qual em grande 
parte é formado pelos outros agentes" (Agostinho, 2003, p.33). Em suma, a adaptação é a característica que permite os sistemas se ajustar às contingencias.

Ao considerar uma organização como um sistema complexo, Stacey (1996) relaciona o conceito de adaptação à aprendizagem organizacional. Segundo o autor, o aprendizado caracteriza-se como um processo auto-organizado capaz de produzir resultados emergentes radicalmente imprevisíveis (Stacey, 1996). Na visão do autor, o processo de aprendizagem pode se dar de forma linear, num processo de aprendizado direto através da informação, ou de forma não linear, no qual o conhecimento adquirido sofre um processo de interiorização e reflexão para depois ser processado em retorno ao meio (Stacey, 1996).

Da mesma forma, Lewin et al (1999) afirma que a organização enquanto um sistema complexo pode se adaptar em respostas as mudanças das condições ambientas de duas formas: a) diante de um ambiente incerto, realizando uma adaptação incremental da forma organizacional; b) ambiente de grande turbulência; gerando novos padrões de organização.
A evolução é consequência do próprio processo de adaptação dos sistemas, Kelly e Alison (1998) identificam diferentes de níveis de adaptação, que correspondem a diferentes estágios evolutivos do sistema. A adaptação leva a um processo de evolução, que é outra característica marcante do sistema complexo, está ligada ao fato de que o sistema não atinge um estado de equilíbrio, mas continua a evoluir; e evolução aqui deve ser entendida a partir da noção de irreversibilidade em um sistema complexo, isto é, a evolução não implica progresso, mas simplesmente que o sistema não pode voltar ao seu estado anterior, mudando sempre para estados que podem ou não ser considerados "melhores" pelos grupos que exercem influência sobre o sistema. A co-evolução significa que todos os subsistemas que influenciam o sistema são também influenciados pelo mesmo, formando uma rede de sistemas em evolução (Murray, 1998).

Em resumo, um sistema complexo pois algumas características constitutivas e de comportamento que os diferenciam de sistemas simples, conforme quadro abaixo:

Quadro1: Síntese dos principais elementos, comportamento e conceitos dos sistemas complexos

\begin{tabular}{|l|l|l|}
\hline Elementos que compõem o sistema complexo & Comportamento do sistema complexo & Conceitos relacionadas \\
\hline $\begin{array}{l}\text { Os agentes que agem localmente e possuem } \\
\text { racionalidade limitada; }\end{array}$ & $\begin{array}{l}\text { Emergem de padrões no nível macro que não } \\
\text { podem ser previstos a partir do comportamento } \\
\text { individual; }\end{array}$ & Emergência/não linearidade \\
\hline $\begin{array}{l}\text { Não há necessariamente equilíbrio e sim um } \\
\text { processo dinâmico de mudança constante com } \\
\text { possíveis períodos mais estáveis; } \\
\begin{array}{l}\text { Os agentes que interagem entre si e com o } \\
\text { (sistema sujeito à influencias ambientais); }\end{array}\end{array}$ & $\begin{array}{l}\text { Os agentes alteram seu comportamento em } \\
\text { resposta ao padrão emergente; }\end{array}$ & Adaptação \\
\cline { 2 - 4 } & $\begin{array}{l}\text { Ocorre um processo evolucionário que seleciona } \\
\text { ou elimina os padrões emergentes; }\end{array}$ & Co-evolução \\
\cline { 2 - 4 }
\end{tabular}

\section{Fonte: Elaborado pelos autores.}

Muitos pesquisadores têm se utilizado dos conceitos da teoria da complexidade para entender e explicar sistemas sociais. A complexidade se aplica a sistemas com agentes múltiplos interagindo e mudando conforme as interações, devido a isso pode ser aplicada à inúmeros tipos de sistemas, inclusive os sociais, pois são compostos por vários membros, que interagem conscientemente com seu ambiente, e entre si (Byrne, 1998; Ciliers, 1988).

Essas características tornam a teoria da complexidade potencialmente aplicável ao estudo do processo político que produz as políticas públicas, na medida em que há múltiplos atores heterogêneos interagindo entre si na disputa por imprimir seus objetivos em determinada política, sendo atingidos por influências diversas e adaptando-se a isso ao longo do tempo (Eppel,2016; Eppel, 2009; Morçol, 2010, Haynes, 2008).

A seguir, tem-se uma breve descrição sobre como a teorização sobre o processo político do qual originam as políticas públicas evoluiu ao longo do tempo, passando a considerá-lo como um processo decisório plural que está sujeito a influência de diversos elementos, como as ideias, interesses e crenças dos atores e as condições contextuais, como as regras do sistema político, por exemplo.

\section{Evolução da Teorização Sobre o Processo Político}

A primeira teorização sobre o processo político veio da aplicação da teoria econômica neoclássica ao mundo da política. $\mathrm{O}$ chamado "modelo racionalista" assumiu que o processo político se resumia ao processo decisório, em que um ator racional (decisor) possuía informações e recursos suficientes, a partir dos quais poderia analisar estrategicamente os problemas e as alternativas e eleger a melhor dentre todas as disponíveis. As políticas públicas seriam resultado da decisão de uma autoridade pública ou de uma autoridade investida de poder público e de legitimidade governamental (Sabatier \& Weible, 2007).

Essa teoria foi duramente criticada nos anos 1940, por Herbert Simon, que ressaltou a necessidade de se incorporar as limitações na capacidade cognitiva do ator, assumindo que o mesmo possui uma racionalidade limitada, operando com informações incompletas, e tomando não a melhor decisão possível, mas sim a mais satisfatória dentro das condições operadas (Simon,1947).

Paralelamente à discussão sobre a natureza do processo das políticas públicas, surgiu a abordagem de Laswell, que entendeu o 
mesmo como sendo formado por fases sequenciais. Neste sentido, o autor poros propôs sete estágios funcionais que se aplicariam ao andamento de qualquer política, que ele denominou: inteligência, promoção, prescrição, invocação, aplicação, rescisão e avaliação (Lasswell, 1951).

Impulsionados pelo trabalho de Lasswell, os modelos que compreendiam as políticas públicas segundo etapas sequenciais, ou estágios, foram bastante populares nas décadas de 1970 e 1980, esses normalmente consideravam que a política pública incluía as fases de formação da agenda, formulação da política, implementação e avaliação. Entretanto, os mesmos foram alvo de críticas por não viabilizar a compreensão de relações causais, sendo imprecisos do ponto de vista descritivo e subvalorizariam o papel da análise e do aprendizado para as políticas públicas (Sabatier, 2007).

Lindblom (1959; 1979) criticou o racionalismo de Laswell e Simon e propôs a incorporação de outras variáveis à análise do processo decisório (Souza, 2006). Neste sentido, assumia que o processo decisório era centralizado, entretanto não se baseava em grandes decisões racionais, a partir da análise das alternativas elencadas e dos custos envolvidos, e sim em decisões "incrementais" sobre as políticas já existentes, pois assim as decisões poderiam ser revertidas a um custo mais baixo se comparadas aos custos de reverter mudanças substanciais (Souza, 2006).

Em meados de 1980 e início de 1990, surgiram algumas teorias procurando compreender o processo político pelo qual originam as políticas públicas, como complexo, instável, e sujeito a relações de poder entre diversos atores. Além disso, passaram a incluir importantes variáveis em suas análises, como as ideias, a mídia, e a opinião pública, considerando-as importantes influências na consolidação de uma política. Dentre esses modelos destacam-se o modelo de Múltiplo Fluxos (Multiples Streams) proposto por John Kingdon em 1984, o de Coalizões de Advocacia (Advocacy Coalitions Framework) proposto por Paul Sabatier e Jekins-Smith em 1993 (posteriormente revisado e ampliado pelos autores em 1999) e o modelo de Equilíbrio Pontuado (Punctuated Equilibrium) elaborado por Frank Baumgartner e Brian Jones em 1993.

O modelo de coalizões de advocacia foi desenvolvido justamente com a preocupação clara de proporcionar uma alternativa para os modelos de análise de políticas públicas baseados em estágios, que, de acordo seus idealizadores, careceriam de robustez conceitual para a construção de hipóteses causais empiricamente testáveis.

Além disso, os autores também estavam preocupados em formular um modelo para estudar as políticas públicas, com base na teoria de sistemas, que sintetizasse algumas das principais contribuições dos debates sobre a gestão top-down e bottom-up; e analisar a influência das informações técnicas nas políticas públicas (Weible, Sabatier \& Mcqueen, 2009).

O modelo, ao enfatizar o papel de valores e ideias, consubstanciados nas crenças das coalizões de defesa, procura construir uma visão geral sobre o funcionamento do subsistema de políticas públicas. Um subsistema seria composto por atores diversificados ativamente preocupados e envolvidos com um problema ou questão política. Pertencem ao subsistema todos os atores que desempenham papéis importantes na formulação e implementação das políticas públicas, e também aqueles que atuam de forma relevante na geração, disseminação e avaliação de ideias relacionadas a elas (Jenkins-Smith \& Sabatier, 1993, 1999).

Externamente ao subsistema de políticas públicas, estariam presentes fatores estáveis bastante difíceis de serem alterados, que podem ser entendidos como fatores estruturais que constrangeriam a ação individual internamente ao subsistema. Estes fatores seriam: os atributos básicos da área do problema, a distribuição dos recursos naturais, os valores socioculturais e estrutura social, e a estrutura de regras básicas do sistema político (Jenkins-Smith \& Sabatier, 1999; Sabatier \& Weible, 2007).

O modelo do equilíbrio pontuado, desenvolvido por Baumgartner e Jones em 1993, também é um exemplo da evolução da teorização em políticas públicas para além do modelo de escolha racional ou de estágios. O modelo inspira-se em elementos da biologia para explicar a ocorrência de longos períodos de estabilidade, interrompidos, ocasionalmente, por rompantes de mudanças abruptas, que marcam a maior parte das políticas públicas (Baumgartner \& Jones, 1993).

O modelo de múltiplos fluxos também surge com o objetivo de proporcionar uma explicação mais abrangente para o processo de formulação de políticas públicas, em especial a fase de construção da agenda governamental, contraponto o modelo sequencial ou de estágios, ele é inclusive considerado o modelo mais completo, na medida em que ao tratar de variáveis diversas - os agentes, ideias, instituições e processos externos - no processo de formulação de políticas públicas, tende a ser uma abordagem mais integradora (John,1998).

O objetivo do modelo é auxiliar na compreensão dos motivos pelos quais um tema passa a chamar a atenção dos formuladores de políticas públicas e ascende à agenda, e porque ao contrário, temas podem deixar a agenda. $\mathrm{O}$ autor do modelo analisou o governo federal norte americano, em uma extensa pesquisa empírica, sendo entrevistados diversos atores no setor de saúde e transporte entre 1976 e 1979. Ele concluiu que o processo de formulação da agenda, é um processo altamente competitivo e que mudanças na agenda ocorrem quando da convergência de três fluxos, os quais possuem uma dinâmica própria e que caminham relativamente independentes: o fluxo político (politics stream), o de soluções (policy stream) e o de problemas (problem stream).

Além da dinâmica dos três fluxos, o autor também ressalta a importância dos diferentes agentes no processo de formulação da agenda, com destaque para a figura do empreendedor de políticas, negociador hábil, que possui a capacidade de unir os três fluxos dando oportunidade para que novos temas ascendam à agenda.

Os três modelos em questão vêm tendo uma extensa aplicação a diferentes realidades políticas, inclusive a brasileira, como demonstram os estudos de Brasil e Capella, (2016) \& Capella, Soares e Brasil (2014). Os três são considerados consagrados no campo de políticas públicas (Petridou, 2014; Schlager \& Weible, 2013) entretanto, outras teorias têm sido apresentadas a partir do 
esforço conjunto de diversos pesquisadores, como demonstram Schlager e Weible (2013), que os consideram como as novas teorias do processo político, que foram apresentados no ano de 2013, na edição especial da revista Policy Studies Journal (Schlager $\&$ Weible, 2013).

A seguir, apresentaremos os procedimentos metodológicos que foram percorridos neste ensaio de forma a cumprir com os objetivos da pesquisa.

\section{Procedimentos metodológicos}

Este ensaio se caracteriza como exploratório e descritivo, bem como busca investigar como as perspectivas teóricas de análise de políticas públicas têm incorporado os conceitos próprios da teoria da complexidade e dos sistemas complexos, mais especificamente os conceitos de emergência, equilíbrio dinâmico, adaptação, e co-evolução (ver quadro 1).

Assim, a ideia é investigar como esses conceitos encontram-se explícita ou implicitamente nessas teorias. Buscou-se comparar as teorias consideradas como "consagradas" (Petridou, 2014; Schlager \& Weible, 2013), desenvolvidas nos anos 1980-1990 quais sejam: o modelo de Coalizões de Advocacia (ACF), Múltiplos Fluxos e Equilíbrio Pontuado, com as novas teorias da área, buscando evidenciar possíveis diferenças entre elas. Além disso, selecionouse as três, pois também são as mais difundidas e aplicadas ao estudo de formulação e implementação de politicas públicas também no Brasil, como demonstram os trabalhos Brasil e Capella, (2016) \& Capella, Soares e Brasil (2014).

Esse recorte comparativo, justifica-se pelas próprias mudanças do processo político dos anos 1980-1990 até os dias atuais, decorrentes das mudanças na própria administração pública, as mudanças de arranjos institucionais, a influência do processo de globalização e a crescente complexidade dos problemas públicos, o que era de se esperar pudesse trazer novos insights teóricos, em que a teoria da complexidade pudesse fazer sentido.

As chamadas novas teorias foram apresentadas em edição especial do Policy Studies Journal e todas elas procuram cada um a sua perspectiva elucidar diferentes aspectos do processo político, são elas: O modelo de ação coletiva institucional (The Institutional Collective Action Framework) proposto por Richard Feiock; o modelo de Ecologia de Jogos (The Ecology of Games Framework) de Mark Lubell; a perspectiva de Regime de Políticas (The Policy Regime Perspective), desenvolvido por Peter J. May e Ashley E. Jochim, o modelo de Robustez (The Robustness Framework), proposto por John M. Anderies e Marco A. Janssen; O modelo de aprendizado coletivo (The Collective Learning Framework), idealizado por Tanya Heikkila e Andrea K. Gerlak; e por último o modelo de Narrativa (The Narrative Policy Framework), fruto do trabalho dos pesquisadores Elizabeth A. Shanahan, Michael D. Jones, Mark K. McBeth, e Ross R. Lane.

Foram escolhidas duas, dentre as seis apresentadas na edição especial do jornal, são elas: o modelo de Ecologia de Jogos (Lubell, 2013) e o modelo de Robustez (Anderies \& Janssen, 2013). A escolha justifica-se pelo fato de que os próprios autores das teorias deixaram claro que entendiam o processo político como um sistema complexo e que as teorias tradicionais não davam conta de explicálo em toda a sua complexidade e que foram elaboradas justamente para superar essa lacuna.

Na seção seguinte, busca-se analisar como a complexidade foi incorporada nas nos modelos teóricos tradicionais, especialmente no Coalizões de Advocacia (ACF), Múltiplos Fluxos e Equilíbrio Pontuado, buscando identificar em cada uma delas se/ ou como os conceitos de emergência, equilíbrio dinâmico, adaptação e coevolução foram abordados explícita ou implicitamente. Em seguida, a mesma análise é feita quanto as novas teorias: o modelo de Robustez e o modelo de Ecologia de Jogos.

\section{Os modelos tradicionais e a complexidade tangenciada}

No modelo de coalizões de advocacia (ACF), o processo político foi caracterizado como um sistema aberto sujeito a trocas com o ambiente e cuja unidade de análise primordial é o subsistema de políticas públicas.

Estes subsistemas - no plural, pois cada subsistema é relativo a uma área específica de políticas públicas - seriam compostos por atores diversificados ativamente preocupados e envolvidos com um problema ou questão política. Todos os atores que desempenham papéis importantes na formulação e implementação das políticas públicas, e também aqueles que atuam de forma relevante na geração, disseminação e avaliação de ideias relacionadas a elas pertenceriam ao subsistema (Sabatier \& Jenkins-Smith, 1999). Esses atores estariam efetivamente envolvidos com o problema e na construção e defesa de alternativas de intervenção (Sabatier, 2007).

Externamente ao subsistema de políticas públicas, estariam presentes os "Parâmetros Relativamente Estáveis", que são fatores estáveis bastante difíceis de serem alterados, mesmo em períodos de uma década ou mais, como por exemplo, os atributos básicos da área do problema, a distribuição dos recursos naturais, os valores socioculturais e estrutura social, e a estrutura de regras básicas do sistema político. Esses fatores seriam responsáveis por criar uma série de constrangimentos à inserção de novas temáticas em agendas governamentais (Sabatier, 2007).

O modelo presta essencial atenção aos momentos de mudanças, estabelecendo hipóteses sobre as condições necessárias para que aconteçam. Os autores consideram que inputs do ambiente externo ao subsistema, a choques internos, acordos negociados e o aprendizado político construído pela interação dos atores no subsistema ao longo do tempo são responsáveis por mudanças no processo de formulação e implementação de políticas públicas (Sabatier \& Weible, 2007).

Eventos externos a um determinado subsistema apresentariam estímulos para geração de mudanças em uma política pública, são exemplos disso, as mudanças em condições sociais, econômicas e políticas; decisões sobre outras políticas públicas; e impactos de outros subsistemas de política.

Os choques internos são perturbações de maior vulto, que impactam nas crenças da coalizão dominante, podendo, portanto, causar a mudança no entendimento a cerca de um problema e sua condução. Os acordos negociados também são apontados como 
um caminho para a mudança, quando não há choques internos ou externos ao subsistema, porém quando há situações de impasse (Sabatier \& Smith, 1999; Sabatier, 2007).

Conforme já discutido, uma característica marcante dos sistemas complexos é o fato de não haver, necessariamente, equilíbrio, e sim um equilíbrio dinâmico, com constantes mudanças. Embora o ACF considere a ideia de sistema aberto sujeito à influências ambientais, momentos de mudança e estabilidade são vistos como momentos distintos, e são "separados" para a explicação do processo político.

Haynes (2009) e Morçöl (2012) argumentam que uma visão coerente com a complexidade não deveria ver mudança e estabilidade como momentos distintos, e sim considerar que o sistema vive em constante mudança, em um equilíbrio dinâmico. O ACF, por sua vez, dá mais ênfase na compreensão da mudança na sua importância enquanto estímulo (que pode mudar a distribuição dos recursos dos atores), que pode influenciar a atuação das coalizões.

Outra característica marcante dos sistemas complexos, é a adaptação. Neste ponto, o modelo parece dialogar implicitamente com a teoria da complexidade, na medida em que considera a necessidade de uma análise da política pública ao longo do tempo, pois de acordo com Sabatier e Jenkins-Smith (1993), o aprendizado orientado para a política afeta os aspectos do subsistema durante extensos períodos de tempo.

Assim, o modelo assume que a interação dos atores dentro do subsistema favoreceria o acúmulo de conhecimento sobre características de um problema e fatores que o afetam, promovendo a avaliação das alternativas adotadas e impactando na realização de mudanças em determinadas políticas públicas (Sabatier \& Smith, 1999; Sabatier, 2007).

Logo, o conceito de "aprendizado orientado a política", remete ao conceito de adaptação dos sistemas complexos, uma vez que o modelo assume que a interação das coalizões, e o papel da informação podem alterar comportamentos a partir da experiência adquirida em determinado problema de política pública.

Ao contrário do ACF, o modelo de múltiplos fluxos preocupa-se com um tipo específico de mudança, ou seja, a mudança na agenda governamental, seja através da inclusão de novos temas ou mesmo ao abandono de temas antes considerados estratégicos.

Neste caso, a unidade de análise não é um sistema ou subsistema e sim "fluxos" que ocorrem de forma paralela e espontânea: o fluxo de problemas, político e de soluções. O fluxo de soluções, ou policy stream "[...] ocorre sem estar necessariamente relacionado à percepção do problema" (Calmon \& Marchesini, 2007, p.8). As alternativas são geradas nas policy communities, ou comunidades geradoras de alternativas. Nelas são geradas as ideias a respeito de soluções, que flutuam em "um caldo primitivo" de políticas, que semelhante ao processo de seleção natural, adapta-se e combinam umas com as outras, permanecem intactas ou ainda são descartadas.

Dessa forma, as alternativas que se mostrem viáveis do ponto de vista técnico e que apresentem custos razoáveis geralmente sobrevivem. A partir deste momento, tais alternativas passam a ser difundidas, não de uma forma automática, mas sim por uma dinâmica de persuasão em que os indivíduos passam a defender a ideia, não só para as comunidades políticas, como também para o público em geral, construindo progressivamente a aceitação da ideia (Kingdon, 2010).

O terceiro fluxo, o político, é composto por elementos tais como: a opinião pública, grupos de pressão, resultados eleitorais, distribuições partidárias ou ideológicas no Congresso e mudanças na administração. Nesse fluxo, três elementos são influentes sobre a agenda governamental: o "humor" nacional (national moodino), as forças políticas organizadas, e as mudanças dentro do próprio governo. O humor nacional, "cria uma espécie de 'solo fértil' para algumas ideias germinarem" (Capella, 2007, p. 29). Assim, o humor favorável pode incentivar a promoção de algumas questões e ao mesmo tempo desestimular a promoção de outras (Capella, 2007).

Os três fluxos mencionados possuem sua dinâmica própria e caminham com relativa independência e por vezes se convergem gerando uma oportunidade de mudança na agenda, a qual Kingdom denomina de janela de oportunidade, ou windows opportunity, que se constituem em momentos transitórios, podendo ocorrer a abertura de "janelas" em determinados momentos bem como o seu fechamento em outros.

Portanto, quando se abre uma janela de oportunidade, é o momento em que uma condição consegue atrair a atenção dos formuladores de políticas públicas, e é também no mesmo momento que há mudanças no fluxo político, as quais permitem mudanças na agenda. E, assim, os formuladores passam a procurar alternativas para os problemas, que já foram desenvolvidas paralelamente no fluxo de soluções. Desta forma, Kingdon aponta que a abertura de janelas de oportunidade é influenciada, sobretudo pelo fluxo de problemas e pelo fluxo político (Capella, 2007).

Para que os três fluxos se liguem, e a janela de oportunidade se abra, é fundamental a figura do policy entrepeneur ou empreendedor de políticas, que são indivíduos que atuam defendendo uma ideia, e agem em momentos políticos oportunos, ligando as ideias a respeito de problemas, às soluções, sendo responsáveis por promover mudanças políticas (Kingdon, 2010; Capella, 2016).

A grande habilidade deste tipo de ator, é perceber o momento oportuno e agir. Estes empreendedores não tem um perfil específico podendo ser atores governamentais ou nãogovernamentais, que investem seus recursos na defesa de propostas com vistas a obtenção de futuros benefícios. Assim, o empreendedor tem um papel central na mudança política, porém não tem poder para sozinho mudar a agenda (Kingdon,2010; Capella, 2016)

A mudança na agenda a partir da abertura de uma janela de oportunidade aproxima-se do conceito de emergência dos sistemas complexos, pois quando um tema ascende à agenda, tem-se então mudanças com relação à agenda anterior. O fenômeno da emergência nos sistemas complexos é caracterizado como uma mudança nos padrões, a partir da emergência de um novo padrão de comportamento do sistema, ao qual os agentes se adaptam e 
que não pode ser previsto se analisado somente o comportamento individual. Da mesma forma, no modelo de múltiplos fluxos, se for analisado somente a dinâmica de cada fluxo, não se pode compreender a abertura de uma janela de oportunidade, e, consequentemente, à ascensão de um tema à agenda.

Embora o policy entrepreneur assuma um papel importante que leva a mudança na agenda, não se pode prever quando irá ocorrer a abertura de uma janela de oportunidade, ou quando irá ocorrer o encontro dos três fluxos.

Da mesma forma o modelo de Equilíbrio Pontuado assemelhase ao de Múltiplos Fluxos, no tocante ao entendimento da dinâmica do processo político. Ao invés de fluxos, o modelo faz referência ao "processamento" de problemas em dois níveis, no nível governamental (macro) e no nível não governamental (micro). As mudanças de pequena envergadura, consideradas incrementais, seriam fruto das decisões tomadas no microssistema ou subsistemas político-institucionais, nos quais as diferentes questões são processadas de forma paralela. Já as mudanças radicais seriam fruto das decisões tomadas no macro sistema político-institucional, onde as questões são tratadas em série (Baumgartner \& Jones, 2010).

O modelo de equilíbrio pontuado assume que muitas questões (policy images) concorrentes sobreviveriam simultaneamente, esperando 0 momento certo para se expandir (Baumgartner \& Jones, 2010), neste ponto também há semelhança com o modelo de Kingdon, que considera que os problemas existem e ao mesmo tempo soluções são elaboradas, independentemente do reconhecimento dos problemas, em outras palavras, soluções e problemas caminhariam de forma paralela e independente.

Em relação à mudança e estabilidade, o modelo propõe que quando uma questão é capturada por um microssistema, tem-se um período de equilíbrio ou quase equilíbrio. Por outro lado, quando uma questão entra na agenda macro política, ocorrem períodos de desequilíbrio, período em que a agenda em políticas públicas pode mudar muito rapidamente, contrariando o consenso de ideias estabelecidas (Baumgartner \& Jones, 2010).

Ao contrário dos microssistemas, os macros sistemas políticos caracterizam-se por intensas e rápidas mudanças, diversos entendimentos sobre uma mesma política (diferentes policy images) e feedback positivo, segundo os autores: "A macro política é a política da pontuação - a política de mudanças em larga escala, das imagens que competem, da manipulação política e da reação positiva" (Baumgartner \& Jones, 2010, p. 137).

Os modelos de múltiplos fluxos e equilíbrio pontuado possuem uma visão processual, e enfatizam a mudança abrupta, significativa, ou, em outras palavras, a emergência de novas questões na agenda governamental.

Os três modelos analisados incorporam alguns conceitos próprios dos sistemas complexos de forma implícita. O ACF traz implícito o conceito de como adaptação (na ideia de aprendizado orientado à política). No MF, destaca-se o conceito de emergência (na ideia de abertura de janela de oportunidade e ascensão de novos temas à agenda) e equilíbrio dinâmico do sistema (em que cada fluxo tem sua própria dinâmica). Da mesma forma que no MF o conceito de emergência também está implícito no modelo de EP (na ideia de imagem capturada por um macrosistema) e equilíbrio dinâmico (ao assumir a existência de dois sistemas, o micro e macro, que operam diferentes questões de forma diferenciada).

Os modelos tradicionais tocam na complexidade de forma tangencial, utilizando-se de alguns conceitos como uma metáfora para explicar determinadas situações do processo político, como a ascensão de temas à agenda. Entretanto, alguns conceitos como a evolução, adaptação são pouco trabalhados.

\section{Novos desenvolvimentos teóricos e a complexidade assumida}

Segundo Petridou (2014), os modelos Robustness Framework, e Ecology of Games Framework possuem em comum o fato de abordarem de diferentes formas a complexidade e incerteza. Nesta seção, buscou-se identificar como tais modelos incorporam explícita ou implicitamente os conceitos de emergência, equilíbrio dinâmico, adaptação e co-evolução

Anderies e Janssen (2013) afirmam que os modelos tradicionais ainda são insuficientes para compreender as políticas públicas em um ambiente cada vez mais complexo, embora reconheçam que avanços tenham sido feitos nesse sentido, mencionando como exemplo, o modelo de coalizões de advocacia de Sabatier e Smith. Entretanto, os autores defendem a necessidade de desenvolvimento de outros ferramentais teóricos, na medida em que o ambiente em que as políticas públicas são construídas é marcado pela fluidez e a globalização.

O modelo de coalizões de advocacia assume que "parâmetros relativamente estáveis", externos ao subsistema, influenciam o processo político e são compostos por fatores que seriam bastante difíceis de serem alterados, como: os atributos básicos da área do problema, a distribuição dos recursos naturais, os valores socioculturais e estrutura social, e a estrutura de regras básicas do sistema político (Sabatier \& Smith, 1999).

Entretanto, Anderies e Jensen (2013) afirmam que na realidade nem sempre isso ocorre, algumas vezes as mudanças nesses parâmetros são mais rápidas que 0 andamento das políticas no subsistema. Eles citam o exemplo da questão das mudanças climáticas, afirmando que os efeitos sentidos no ambiente são mais rápidos (derretimento de geleiras, movimentos sociais ou ambos) do que o andamento da construção de uma política climática, visto que poucas são as evoluções das negociações internacionais do clima.

Portanto, nem sempre o pressuposto de mudanças lentas e parâmetros estáveis do sistema, assumidas pelo modelo de coalizões de advocacia, acontece na realidade. Argumentam então que é necessário que haja uma abordagem sistemática para entender a interação dinâmica entre o que denominam de "contexto político" - equivalente a noção de "parâmetros relativamente estáveis" no modelo ACF - e os "processos de intervenção" equivalente às ações desenvolvidas nos subsistemas de políticas, no modelo ACF (Anderies \& Janssen, 2013). Ao se analisar o modelo, pode-se inferir que os processos de intervenção representariam o espaço de atuação dos atores (micro) e o contexto político (macro), representaria os aspectos estruturais que 
influenciam o comportamento dos atores. Ao fazer essa distinção, os autores parecem tentar romper com a dicotomia entre agencia e estrutura tal qual propõe Morçol (2010)

O modelo de Robustez é uma extensão do modelo de Análise Institucional e Desenvolvimento (IAD) de Ostrom (2009), e pretende compreender questões chave no contexto de gestão de recursos naturais. Os atores deixam explícito que a política pública neste caso, é entendida como um componente de um sistema adaptativo complexo, que denominam de Sistema sócio-ecológico (SES), no qual a política afeta e é afetada pelos processos biofísicos do contexto a qual está inserida. Exemplos desses processos biofísicos seriam a degradação do solo, alterações climáticas entre outros (Anderies \& Janssen, 2013).

O sistema sócio-ecológico é composto de um conjunto de atores (usuários de recursos), uma estrutura de governança, e um sistema de recursos, que estão continuamente evoluindo e adaptando-se em diferentes escalas e níveis do sistema. Assim, o próprio processo politico é visto como propriedade emergente de um sistema sócio-ecológico dinâmico. A estrutura desses relacionamentos é o que os autores denominam de "aptidão" entre as condições biofísicas, atores, e as regras. Dessa forma, um sistema com boa aptidão é aquele que aproveita estruturas biofísicas e sociais para reduzir os custos de monitoramento, de sanção e de gestão de conflitos (Anderies \& Janssen, 2013).

Os autores defendem que embora não seja possível compreender todos os aspectos de um sistema complexo, os pesquisadores precisam aprender a como gerenciá-los. Alertam para o fato de que em um mundo em constante mudança as políticas que são apropriadas para um contexto podem revelar vulnerabilidades em outro (Anderies \& Janssen, 2013).

A experimentação seria um mecanismo para aumentar a probabilidade de alcançar uma boa adequação entre as políticas e as condições locais, o que poderia ser feito por meio do estudo de grandes sistemas a fim de identificar quais os aspectos são robustos ao tamanho do sistema e aqueles que não são. A ideia é se chegar a um ponto ótimo entre vulnerabilidade e robustez (Anderies \& Janssen, 2013). Entretanto, os autores não propõem qual seria a metodologia adequada para se fazer isso.

Os autores defendem que não se deve pensar em processo políticos "direcionados por coalizões que defendem a 'política certa' e sim que devem ser pensados direcionamentos que estimulem a experimentação, adaptação e aprendizado" (Anderies \& Janssen, 2013, p.532).

O modelo assume claramente que entende o processo político como fundamentalmente complexo, principalmente ao se considerar as mudanças rápidas promovidas pela globalização. Ao considerar a interação dinâmica entre "contexto político" e os "processos de intervenção", o modelo assume uma das características de um sistema complexo, que é a constante interação entre as partes em um equilíbrio dinâmico. Entretanto, o modelo não aborda claramente a questão da evolução nem da emergência, entendendo como características naturalmente esperadas da interação entre as duas dimensões, porém sem fazer referencias explícitas às mesmas. Da mesma forma, a ideia de aptidão do sistema, leva a concluir que a evolução é uma característica do sistema como um todo, entretanto o modelo não deixa claro como a evolução seria operacionalizada.

A ideia de gerenciamento do sistema é uma das razões para a proposição do modelo, pois segundo os autores, mesmo não se podendo conhecer todo o funcionamento de um sistema complexo, devido a incerteza, é possível gerenciá-lo. Vale ressaltar também, que os próprios autores não se preocupam em explicar o que seria esse "gerenciamento do sistema".

Quanto ao modelo de Ecologia de Jogos, Lubell (2013, p. 357), afirma, claramente, que "a complexidade institucional não é uma hipótese, é um fato" e que as ciências políticas precisam de um modelo teórico para analisar empiricamente "a estrutura, processo, evolução e resultados de tais sistemas adaptativos complexos" (Lubell, 2013, p.537). Visando preencher essa lacuna, o autor atualizou o modelo de Ecologia dos Jogos (EG) desenvolvido por Norton Long (1958).

A principal característica do modelo de Ecologia de Jogos é proporcionar a análise da interação de diversas políticas simultaneamente (governança), considerando que o resultado de uma política se dá em função de decisões tomadas em múltiplos "jogos" ao longo do tempo. Um jogo político consiste em um conjunto de atores políticos que participam de um processo de tomada de decisão coletiva regido por regras formais ou informais - que denomina de "instituição política" - que ocorre dentro de uma arena política geograficamente definida - denominada "sistemas" (Lubell, 2013).

Os sistemas de política são territórios com problemas públicos, múltiplas instituições relativas ao problema, e vários atores. Os sistemas podem ser definidos em diferentes escalas: local, regional, estadual, nacional e global. Parte-se do pressuposto que as instituições estão ligadas em vários níveis, e uma decisão em uma instituição afeta o conjunto de possíveis soluções de outra instituição (Lubell, 2013). Por exemplo, uma decisão a nível de federal, irá moldar a dinâmica da "ecologia política" em níveis mais baixos, como o estadual e o municipal.

Os resultados das políticas dependem ainda de como os indivíduos tomam decisões a respeito do uso dos recursos em cada questão. Os atores também podem se envolver em comportamento estratégico em diferentes sistemas, do local ao global, a fim de reformular as instituições em diferentes níveis para melhor prosseguir os seus interesses (Lubell, 2013).

A mudança no jogo pode ocorrer induzida por fatores internos ou externos. A primeira, ocorre a partir das estratégias dos próprios atores envolvidos, que podem transitar por diferentes escalas, e envolvem-se em processos de aprendizado político, podendo criar e destruir instituições. A segunda forma, pode decorrer da mudança nos recursos existentes e advir de decisões em instituições de nível superior, que impactariam nas decisões no jogo local. As mudanças seriam de difícil previsão e, às vezes, poderiam ser expressivas. O sistema que consegue se adaptar ao ambiente em constante mudança e continuar a promover soluções para problemas de ação coletiva ao longo do tempo, seria considerado eficiente ou resiliente (Lubell, 2013). 
Lubell (2013) assume, claramente, que se baseou na literatura dos sistemas complexos adaptativos para propor o modelo, assumindo como pressuposto que os padrões globais do sistema são resultado de inúmeras interações locais entre os componentes heterogêneos e de auto-organização dos atores em diferentes escalas.

O componente da evolução do modelo estaria representado pela sobrevivência das instituições, as quais dependem de apoio político dos atores. Assim, a probabilidade de sobrevivência das instituições, estaria relacionada à sua eficácia, que é entendida como a habilidade de resolução de problemas de ação coletiva e de distribuição de recursos de forma que os atores poderosos percebam como justa (Lubell, 2013).

O modelo pretende produzir hipóteses empiricamente testáveis sobre a estrutura e função dos sistemas que envolvem a interdependência de várias políticas, os processos que influenciam o comportamento individual e a mudança institucional, bem como compreender como diferentes tipos de arranjos institucionais estão ligados aos resultados e os efeitos das políticas, a fim de fornecer recomendações sobre como gerenciar o sistema. Para o autor, os acadêmicos e analistas de políticas devem fornecer recomendações aos policymakers a fim de ajudá-los a alcançar seus objetivos, e o modelo proposto teria essa função (Lubell, 2013).

A noção de interação entre os componentes, que é central ao conceito de sistema complexo, aqui é mais alargada que no modelo de Robustez, pois além de compreender a interação entre o "processo político" e o "contexto político", o modelo considera a interação sobre decisões políticas em diversos níveis como fatores causais para a explicação de determinada política. Isto porque as instituições são consideradas interligadas. Neste ponto, o modelo se propõe a fazer tanto uma análise longitudinal, como também das interações verticais (entre os diferentes níveis, do internacional ao local).

Da mesma forma que no modelo de Robustez em que um sistema é considerado adequado, neste caso, o sistema também é considerado eficiente ou resiliente de acordo com sua capacidade de adaptação e de continuar a promover soluções eficazes para os problemas. As instituições são os componentes em que a evolução operaria, na medida em que aquelas consideradas mais eficazes do ponto de vista da resolução dos problemas sobreviveriam ao longo do tempo. Da mesma forma, o autor justifica que a ideia de se compreender um sistema complexo é fundamentalmente gerenciálo.

Em suma, o conceito de equilíbrio dinâmico está implícito na ideia de interação dinâmica entre o "contexto político" e os "processos de intervenção", do modelo de Robustez. O modelo não aborda a questão da evolução, seu foco está em compreender as interações dos componentes em constante mudança, o que de certa forma traz imbuída a ideia de adaptação. Entretanto, o modelo não aborda claramente a questão da evolução nem da emergência, sem fazer referencias explícitas às mesmas.

O modelo de Lubell (2013) parece abarcar todos os conceitos de um sistema complexo, sendo a interação para ele mais detalhada que no modelo de Robustez. A unidade de análise é alargada para além dos dois níveis do modelo de Anderies e Janssen (2013), assumindo que o processo político ocorre em diferentes níveis desde o internacional ao local, e essa interação influencia na política formulada e implementada. Dessa forma, além da interação longitudinal, ao longo do tempo, o modelo, também se presta a analisar a interação vertical (entre os níveis).

A noção de adaptação também está presente ao considerar que um sistema poderia ser mais ou menos resiliente ou eficiente na medida em que consegue em maior ou menor grau se adaptar às constantes mudanças. As instituições, nesse caso, seriam o componente em que a evolução opera, na medida em que o modelo considera que as instituições mais eficazes evoluiriam. Da mesma forma que no modelo de Robustez, a noção de gerenciamento do sistema também se faz presente.

Entretanto, os dois modelos não propõem uma metodologia específica para se conduzir a análise conforme seus pressupostos. Lubell (2013) sugere que a análise de redes sociais pode contribuir, entretanto, assume que outros direcionamentos metodológicos ainda precisam ser pensados.

\section{Considerações finais}

A partir da discussão empreendida, pôde-se evidenciar que que as novas teorias analisadas possuem a preocupação explícita de incorporar os conceitos da complexidade, diferentemente das teorias tradicionais que não possuíam tal preocupação, ou, pelo menos, não era uma preocupação explicita ao leitor. Nas teorias tradicionais, os conceitos próprios dos sistemas complexos apareciam implícitos na construção dos modelos, com uso quase que metafórico para explicar determinados fenômenos do processo político, como a mudança na agenda e o aprendizado dos atores. Entretanto, não foram oferecidas ferramentas que pudessem capturar essa interdependência dinâmica entre os agentes e a estrutura.

Nas novas teorias analisadas, a interdependência dinâmica entre agente e estrutura aparece mais explicitamente, a partir da ideia de interação entre "contexto político" e "processos de intervenção" (no modelo de Robustez) e entre instituições políticas" e "sistemas" (no modelo de Ecologia de Jogos). O modelo de Robustez, assim como os modelos tradicionais, não incorporou outros aspectos importantes da complexidade, como emergência e evolução. Nesse sentido, o modelo de Ecologia dos Jogos parece mais completo, incorporando os principais conceitos do sistema complexo, como equilíbrio dinâmico, emergência, adaptação, coevolução,

Entretanto, as novas teorias não apresentam as metodologias de análise, ou seja, não explicam como seria possível analisar essas interações. Talvez porque o objetivo não seja compreender essas interações, mas sim escolher a melhor solução, a que torne o sistema mais eficiente. Percebem-se, nessas novas teorias, que está implícito uma visão de formulação de políticas públicas com um caráter mais tecnicista, que parece deixar de lado, o conflito, a argumentação, e a própria política do processo político. Exemplo disso, é o fato de que os modelos assumem a que experimentação 
pode ser capaz de encontrar uma boa adequação entre política e condições locais, identificando fatores que são robustos ou vulneráveis no sistema. E que é possível gerenciar esse sistema (como afirma o modelo de Robustez), e/ou fornecer conselhos aos tomadores de decisão, como propõe o modelo de Ecologia de Jogos.

Assim, pela analise aqui empreendida, é possível supor que uma maior incorporação ou não de conceitos da complexidade, ou em outras palavras, a complexidade assumida ou tangenciada, tem razões explicativas no próprio entendimento do que vem a ser o processo político sob o qual as políticas públicas são formuladas e implementadas, e do papel que a análise de políticas públicas deve cumprir: uma função prescritiva ou descritiva.

A análise empreendida permitiu visualizar um movimento em direção a um esforço para se pensar outras abordagens teóricas e metodológicas, capazes de compreender o processo de políticas públicas, caracterizados pelas constantes mudanças, incertezas e instabilidades. Entretanto, não existe uma única perspectiva ao se tentar analisar o processo político como um sistema complexo, visto que 0 próprio conceito de complexidade possui múltiplas interpretações. Os pesquisadores do campo de análise políticas públicas parecem encontrar-se neste engajamento diante de uma realidade cada vez mais complexa.

\section{Referências}

Agar, M. (1999). Complexity theory: An exploration and overview based on John Holland's work. Field Methods, 11(2), 99-120.

Agostinho, M. C. E. (2003). " Administração Complexa": Revendo as Bases Científicas da Administração. RAE-eletrônica, 2(1).

Anderies, J. M., \& Janssen, M. A. (2013). Robustness of Social-Ecological Systems: Implications for Public Policy. Policy Studies Journal, 41(3), 513536.

Baranger, M. (2000). Chaos, complexity, and entropy. New England Complex Systems Institute, Cambridge.

Baumgartner, F. R., \& Jones, B. D. (1993). Punctuated equilibria in politics. Agendas and instability in American politics, 3-24.

Baumgartner, F. R., \& Jones, B. D. (2010). Agendas and instability in American politics. University of Chicago Press.

Bousquet, A., \& Curtis, S. (2011). Beyond models and metaphors: complexity theory, systems thinking and international relations. Cambridge review of international affairs, 24(01), 43-62.

Brasil, F. G., \& Capella, A. C. N. (2016). Os estudos das políticas públicas no Brasil: passado, presente e caminhos futuros da pesquisa sobre análise de políticas. Revista Política Hoje, 25(1), 71-90.

Budd, L., Charlesworth, J., \& Paton, R. (2006). Making policy happen. Routledge.

Byrne, D. S. (1998). Complexity theory and the social sciences: an introduction. Psychology Press.

Cairney, P. (2012). Complexity theory in political science and public policy. Political Studies Review, 10(3), 346-358.

Calmon, P. C. D. P., \& Costa, M. M. (2007). Análise de Políticas Públicas no Brasil: estudos sobre a formação da agenda governamental. Brasília, DF.

Capella, A. C. N. (2007). Perspectivas teóricas sobre o processo de formulação de políticas públicas. Políticas públicas no Brasil. Rio de Janeiro: Fiocruz, 1, 87-124.

Capella, A. C., \& Soares, A. G.; Brasil, F.G. (2014). Pesquisa em Políticas Públicas no Brasil: Um mapeamento da aplicação de modelos internacionais recentes na literatura nacional. ENCONTRO DA ABCP, 9

Cilliers, P. (2005). Complexity, deconstruction and relativism. Theory, Culture \& Society, 22(5), 255-267.

Cohen, M. D., \& Axelrod, R. (2000). Harnessing complexity: organizational implications of a scientific frontier. Simon and Schuster.

Eppel, E. A. (2009). The contribution of complexity theory to understanding and explaining policy processes: A study of tertiary education policy processes in New Zealand.

Eppel, E. (2016). Complexity thinking in public administration's theories-inuse. Public Management Review, 1-17.

Ferlie, E., Lynn, L. E., \& Pollitt, C. (2005). The Oxford handbook of public management. Oxford University Press, USA.
Gatrell, A. C. (2005). Complexity theory and geographies of health: a critical assessment. Social Science \& Medicine, 60(12), 2661-2671.

Gerrits, L., \& Marks, P. (2015). The evolution of Wright's (1932) adaptive field to contemporary interpretations and uses of fitness landscapes in the social sciences. Biology \& Philosophy, 30(4), 459-479.

Geyer, R. \& Rihani, S. (2010). Complexity and public policy: a new approach to twenty-first century politics, policy and society. Routledge.

Giddens, A. The Constitution of Society. Cambridge, UK: Polity Press, 1984.

Goulielmos, A. M. (2005). Complexity theory: a science where historical accidents matter. Disaster Prevention and Management: An International Journal, 14(4), 533-547.

Haken, A., \& Cook, S. A. (1999). An exponential lower bound for the size of monotone real circuits. Journal of Computer and System Sciences, 58(2), 326-335.

Haynes, P. (2009, abril). Complexity in Public Policy: Metaphors and Methods. Aula inaugural na Universidade de Brighton. Recuperado de: https://www.youtube.com/watch?v=3VHFUd3HQDU

Haynes, P. (2008) Complexity theory and evaluation in public management: a qualitative systems approach. Public Management Review, 10 (3). pp. 401 419.

Holland, J. H (1995). Hidden order: How adaptation builds complexity. Basic Books.

Horgan, J. (1995). From complexity to perplexity. Scientific American, 272, 104-109.

Jenkins-Smith, H. C., \& Sabatier, P. A. (Eds.). (1993). Policy change and learning: an advocacy coalition approach. Westview press.

Jenkins-Smith, H. C., \& Sabatier, P. A. (1999). The advocacy coalition framework: an assessment. Theories of the policy process. Westview Press, Boulder, 117-166

John, P. (1998). Analysing Public Policy. A\&C Black.

Kauffman, S. A. (1993). The origins of order: Self-organization and selection in evolution. Oxford University Press, USA.

Kelly, S.\& Allison, M. A. (1998). The complexity advantage: how the science of complexity can help your business achieve peak performance. New York.

Kingdon, J. W. (2010). Agendas, alternatives and public policies (2nd ed.). New York: Addison-Wesley.

Klijn, E. H. (2008). Complexity Theory and Public Administration: What's New? Key concepts in complexity theory compared to their counterparts in public administration research. Public Management Review, 10(3), 299-317.

Koliba, C. (2014). Governance network performance: A complex adaptive systems approach. In Keast, R.; Mandell, M. \& Agranoff, R. (Eds.), Network theory in the public sector: Building new theoretical frameworks. (pp. 84-102). London: Routledge.

Koppenjan, J. F. M., \& Klijn, E.-H. (2014). What can governance network theory learn from complexity theory? Mirroring two perspectives on complexity. In Keast, R., Mandell, M. \& Agranoff, R. (Eds.), Network theory in the public sector: Building new theoretical frameworks. (pp. 157-173). London: Routledge.

Lasswell, H. D. (1951). The Immediate Future of Research Policy and Method in Political Science. American Political Science Review, 45(01), 133-142.

Leite, M. S. A. (2004). Proposta de uma modelagem de referência para representar sistemas complexos.

Lewin, A. Y., Long, C. P., \& Carroll, T. N. (1999). The coevolution of new organizational forms. Organization science, 10(5), 535-550.

Long, N. E. (1958). The local community as an ecology of games. American Journal of Sociology, 251-261.

Louth, G., Mitzenmacher, M., \& Kelly, F. (1994). Computational complexity of loss networks. Theoretical Computer Science, 125(1), 45-59.

Lubell, M. (2013). Governing institutional complexity: The ecology of games framework. Policy Studies Journal, 41(3), 537-559.

Mainzer, K. (1993). Philosophical foundations of nonlinear complex systems. In Interdisciplinary approaches to nonlinear complex systems (pp. 32-43). Springer Berlin Heidelberg.

Manson, S. M. (2001). Simplifying complexity: a review of complexity theory. Geoforum, 32(3), 405-414.

Maturana, H. R., \& Varela, F. J. (1991). Autopoiesis and cognition: The realization of the living (Vol. 42). Springer Science \& Business Media.

Mitleton-Kelly, E. (2003). Complex systems and evolutionary perspectives on organisations: the application of complexity theory to organisations. Elsevier Science Ltd.

Moran, M., Rein, M., \& Goodin, R. E. (2006). The Handbook of Public Policy. Morin, E. (2007). O Método 1: a natureza da natureza. Porto Alegre: Sulina 2003. NANI. Vereda tropical. Estado de Minas, Caderno de Cultura, 6.

Morçöl, G. (2002). A new mind for policy analysis: Toward a post-Newtonian and postpositivist epistemology and methodology.

Morçöl, G. (2005). A new systems thinking: implications of the sciences of complexity for public policy and administration. Public Administration Quarterly, 297-320. 
Morçöl, G. (2010). Issues in reconceptualizing public policy from the perspective of complexity theory. Emergence: Complexity and Organization, 12(1), 52.

Morçöl, G. (2012). Urban Sprawl And Public Policy: A Complexity Theory Perspective. Emergence: Complexity and Organization, 14(4), 1.

Murray, P. J. (1998). Complexity theory and the fifth discipline. Systemic Practice and Action Research, 11(3), 275-293.

Ostrom, E. (2009). Beyond markets and states: polycentric governance of complex economic systems. Transnational Corporations Review, 2(2), 1-12.

Petridou, E. (2014), Theories of the Policy Process: Contemporary Scholarship and Future Directions. Policy Stud J, 42: S12-S32.

Prigogine, I. (1987). Exploring complexity. In G. Midgley (Ed.), Systems Thinking (pp. 409-417). London: Sage.

Prigogine, I. (1996). O fim das certezas. Unesp.

Puddifoot, J. E. (2000). Some problems and possibilities in the study of dynamical social processes. Journal for the Theory of Social Behaviour, 30(1), 79-97.

Rhodes, M. L., Murphy, J., Muir, J., \& Murray, J. A. (2011). Public management and complexity theory: Richer decision-making in public services. New York: Routledge.

Sabatier, P. A. (2007). Theories of the Policy Process. 2. ed. Boulder: Westview Press.

Sabatier, P., \& Weible, C. (2007). The advocacy coalition: Innovations and clarifications. Theories of the policy process, 2, 189-220.
Sanger, M., \& Giddings, M. M. (2012). A simple approach to complexity theory. Journal of Social Work Education, 48(2), 369-376.

Schlager, E., \& Weible, C. M. (2013). New theories of the policy process. Policy Studies Journal, 41(3), 389-396.

Simon, H. A. (1947). Administrative behavior; a study of decision-making processes in administrative organization.

Souza, C. (2006). Políticas públicas: uma revisão da literatura.

Stacey, R. D. (2007). Strategic management and organisational dynamics: The challenge of complexity to ways of thinking about organisations. Pearson education.

Teisman, G., \& Klijn, E.-H. (2008). Complexity theory and public management: An introduction. Public Management Review, 10(3), 287-297.

Teisman, G., Van Buuren, A., \& Gerrits, L. (Eds.). (2009). Managing complex governance systems: Dynamics, self-organization, and coevolution in public investments. New York: Routledge.

Urry, J. (2003). Global complexity (pp. 51-59). Cambridge: Polity. Wu, J., \& Marceau, D. (2002). Modeling complex ecological systems: an introduction. Ecological Modelling, 153(1), 1-6.

Weible, C. M., \& Sabatier, P. A. (2005). Comparing policy networks: Marine protected areas in California. Policy Studies Journal, 33(2), 181-201.

Weible, C. M., Sabatier, P. A., \& McQueen, K. (2009). Themes and variations: Taking stock of the advocacy coalition framework. Policy Studies Journal, 37(1), 121-140. 